\title{
Phylogenetic analyses of nitrogen-fixing cyanobacteria from the Baltic Sea reveal sequence anomalies in the phycocyanin operon
}

Department of Biology and Environmental Science, University of Kalmar, Barlastgatan 1, S-391 82 Kalmar, Sweden

\author{
Sven Janson and Edna Granéli
}

Author for correspondence: Sven Janson. Tel: +46 480447310 . Fax: +46 480447305 . e-mail: sven.janson@hik.se

The examination of molecular phylogenies of cyanobacteria and other microorganisms is increasing dramatically. The use of a single locus in these studies leaves the resulting phylogenies unconfirmed. In this study, the partial sequences of two loci containing segments of protein-encoding genes, the het $R$ and the phycocyanin locus (PC-IGS), were examined. Laboratory strains and natural populations of the heterocyst-forming cyanobacteria Anabaena, Aphanizomenon and Nodularia from the Baltic Sea were used, in total 41 sequences were determined and their phylogenies were analysed with maximum-likelihood methods. The het $R$ phylogenies suggested that the planktonic Aphanizomenon and Nodularia each comprise one species, while there were numerous Anabaena species present in the Baltic Sea. In the case of Nodularia, the PC-IGS phylogenies were incongruent with this and suggested that several lineages of Nodularia plankton species existed. In the hetR phylogeny, the floating and nodularin-producing strains of Nodularia were grouped together. For both the hetR and PC-IGS loci of cultured species of Nodularia their molecular phylogeny did not correspond well with the affiliation suggested by morphology. In sequences derived from species of Anabaena and Aphanizomenon the PC-IGS and hetR phylogenies were congruent, suggesting that Aphanizomenon sp. from the Baltic Sea is genetically distinct from both Aphanizomenon flos-aquae from lakes and Aphanizomenon sp. TR183 from the Baltic Sea. In both Nodularia and AnabaenalAphanizomenon, the PC-IGS sequences showed a significant degree of either recombination events or selection, while none was detected within the het $R$ sequences. This is the first study comprising the phylogenies of multiple loci from all heterocystous cyanobacteria from the Baltic Sea and shows that earlier results using the PC-IGS locus should be interpreted cautiously in the absence of a confirmation using a second locus.

Keywords: Anabaena, Aphanizomenon, het $R$, Nodularia, phycocyanin

\section{INTRODUCTION}

The cyanobacteria are diverse in habitat and mor-

Abbreviations: PCC, Pasteur Culture Collection; KAC, Kalmar Algae Collection; BC Nod, Bristol Collection Nodularia; PC-IGS, the intergenic transcribed spacer between, and flanking regions of $c p c B$ and $c p c A$.

A table of the taxa and accession numbers used in this study is available as supplementary data in IJSEM Online (http://ijs.sgmjournals.org).

The GenBank accession numbers for the hetR sequences determined in this study are AF320004-AF320008 and AF364336-AF364338. The GenBank accession numbers for the sequences of PC-IGS determined in this study are AF364339-AF364343, AY036900, AY036901 and AF193249. phology but the genetic diversity does not always conform to the phenotypic diversity. Nitrogen fixation by filamentous cyanobacteria in the oceans plays an important role in the global nitrogen budget (Capone et al., 1997; Carpenter et al., 1999). In the Baltic Sea, species of the heterocyst-forming cyanobacteria Aphanizomenon Morren, Nodularia Mertens and Anabaena Bory become the dominant nitrogen-fixing cyanobacteria and fix $\mathrm{N}_{2}$ in the order of $10^{5}$ tonnes $\mathrm{N}$ per year (Leppänen et al., 1988; Rahm et al., 2000). There are several unresolved taxonomic questions regarding the Baltic Sea cyanobacteria. The Aphani- 
zomenon sp. in the Baltic Sea is often referred to as Aphanizomenon flos-aquae but ultrastructural observations have challenged this view (Janson et al., 1994). Several planktonic species of Nodularia from the Baltic Sea have been described based on ultrastructure and morphology, including $N$. baltica, $N$. litorea and $N$. spumigena (Komárek et al., 1993). In a study using both phenotypic and genetic analysis of species of Nodularia it was suggested that only one species exists in the Baltic Sea (Barker et al., 1999). These genera have been subjected to intense phylogenetic studies using gene sequences, RFLP and fingerprinting methods (Neilan et al., 1995; Bolch et al., 1999; Barker et al., 2000a; Lehtimäki et al., 2000; Lyra et al., 2001; Moffitt et al., 2001). None of these studies used gene sequences from more than one locus to be able to confirm the observed phylogenies and in the absence of confirmation, the resulting phylogenies are open to question.

The assessment of the phylogeny of organisms through gene sequence analysis has increased dramatically since the advent of PCR and automated sequencing. Most studies have used the 16S rRNA gene for molecular phylogenies and inference of bacterial evolution. The variation of the $16 \mathrm{~S}$ rDNA sequences between closely related species of Bacillus have been found to be insufficient to separate them (Fox et al., 1992). In filamentous planktonic cyanobacteria, the $16 \mathrm{~S}$ rDNA has been found to vary less than proteinencoding gene sequences (Janson et al., 1999a; Barker et al., 2000a, b). Two loci comprising partial proteinencoding genes that have been used to infer phylogenies of cyanobacteria are the het $R$ gene (Janson et $a l ., 1999 \mathrm{a}, \mathrm{b}$ ) and the region including the intergenic space and flanking regions of $c p c B$ and $c p c A$ genes (PC-IGS) (Bolch et al., 1999; Barker et al., 2000a; Robertson et al., 2001; Tillett et al., 2001). The het $R$ gene encodes a serine-type of protease with a regulatory role in the differentiation process of heterocysts (Zhou et al., 1998; Buikema \& Haselkorn, 2001). The het $R$ gene has only been detected in filamentous cyanobacteria (Buikema \& Haselkorn, 1991; Janson et al., 1998), which facilitates the analysis of samples containing other micro-organisms. The phycocyanin genes $c p c B$ and $c p c A$ encode proteins making up the accessory pigments of photosystem II (Gantt, 1994). The PC-IGS locus is found in some photosynthetic organisms including cyanobacteria, red algae and cryptophytes. Since the PC-IGS locus contains both coding and non-coding regions one could expect that different parts of the sequence would give different phylogenies, i.e. different evolutionary constraints exists for non-coding and coding regions (Li \& Grauer, 1991). Despite this, many phylogenetic analyses have been performed using PC-IGS, without addressing the possibility of sequence anomalies within this locus.

In this study, datasets including two loci from identical or comparable strains were used. The phylogeny and sequence anomalies of the het $R$ and PC-IGS loci from heterocyst-forming cyanobacteria from the Baltic Sea were analysed. The results showed that the phylogenies of these two loci were to some extent incongruent, and sequence anomalies were detected in the PC-IGS locus but not in the het $R$ locus.

\section{METHODS}

Organisms. The cyanobacterial isolates that were examined by sequencing in this study are all maintained in the Kalmar Algae Collection (KAC) at the University of Kalmar, Sweden, except Aphanizomenon sp. TR183, which was obtained from Dr K. Sivonen, Department of Applied Chemistry and Microbiology, Helsinki University, Finland. Strains $N$. spumigena KAC 10 through 17, Anabaena lemmermannii KAC 16 and Aphanizomenon sp. KAC 15 were isolated from the sound between Öland and the mainland of Sweden. Nodularia spp. KAC 64 through 68 and Aphanizomenon sp. KAC 60 were isolated from the Landsort deep in the Northern Baltic proper station number BY $31\left(58^{\circ} 35^{\prime} \mathrm{N}, 18^{\circ} 14^{\prime} \mathrm{E}\right)$. All cultures were maintained at $16{ }^{\circ} \mathrm{C}$, under cool fluorescent light tubes giving a light intensity of $30 \mu \mathrm{mol} \mathrm{s}^{-1} \mathrm{~m}^{-2}$, and grown in a Baltic Sea water-based f/2 medium with silicate omitted (Rippka, 1988). The natural population of Aphanizomenon sp. A1, A5 and the water used in mesocosm experiments where the Anabaena spp. were collected originated from an area 6 nautical miles east of Öland in the central part of the Baltic proper. Aphanizomenon sp. NB1 and NB5 were sampled at station BY 31. The natural population of Aphanizomenon flos-aquae was collected in lake Bysjön, Scania, Sweden, and the one of Aphanizomenon flos-aquae var. klebahnii originated from a park lake, Lilla Pildammen, Malmö, Sweden.

The online version of this paper (at http://ijs.sgmjournals. org) contains a supplementary table of the taxa and accession numbers used in this study. The assignments of morphospecies were done according to the descriptions in Geitler (1932).

PCR amplification and sequencing. Filaments or colonies of filaments, from natural populations were transferred to PCR tubes in a volume of five $\mu$ l of environmental water. DNA was extracted using the procedure described earlier (Janson et al., 1999a), briefly the cells are lysed by lysozyme, SDS and heat treatment. The DNA was then extracted from the crude lysate with cetyl tri-methyl ammonium bromide (CTAB). The 448-bp fragment of het $R$, corresponding to numbers 159-606 in Nostoc sp. PCC 7120 (Buikema \& Haselkorn, 1991), was amplified from extracted DNA or intact filaments using primers hetr1 and hetr2 (Janson et al., 1998). The PC-IGS region was amplified using primers PCBF and PCAR (Neilan et al., 1995). The PCR reaction mix was supplemented with $2 \cdot 5 \%(\mathrm{v} / \mathrm{v})$ final concentration of DMSO (Sigma) in order to lower the annealing temperature and improve the permeability of the cells. The reaction mix was also supplemented with Taq DNA polymerase buffer (Amersham Pharmacia Biotech), $200 \mu \mathrm{M}$ dNTP (Amersham Pharmacia Biotech) and 1.5 U Taq DNA polymerase. The PCR was run for 30 cycles with $60 \mathrm{~s}$ at $94{ }^{\circ} \mathrm{C}, 30 \mathrm{~s}$ at $45^{\circ} \mathrm{C}$ and $30 \mathrm{~s}$ at $72{ }^{\circ} \mathrm{C}$, followed by an extension of $7 \mathrm{~min}$ at $72^{\circ} \mathrm{C}$. The PCR products used in sequencing were purified with QIAquick PCR purification kit (Qiagen). The purified PCR products were sequenced bidirectionally using an ABI model 373A automated sequencer (Applied Biosystems) and dye-terminator chemistry, using the same primers, hetr1, hetr2, PCBF and PCAR. When needed, sequencing was repeated on independent 
PCR products to exclude errors incorporated by Taq polymerase or in the sequencing reaction (von Wintzingerode et al., 1997).

Sequence analysis. The GenBank accession numbers for the sequences determined in this study and those used for phylogenetic analyses are listed in the online version of this paper (at http://ijs.sgmjournals.org). Alignments of sequences were produced manually with Seaview (Galtier et al., 1996). The trees were constructed using the PhyloWin package (Galtier et al., 1996) and PHYLIP 3.6 [http:// evolution.genetics.washington.edu/phylip.html]. The sequences from Nostoc sp. PCC 7120 were used as outgroups in all trees. When using distance matrices to generate the trees the correction of Jukes \& Cantor (1969) was used. When using maximum-likelihood in PhyloWin the fastDNAml (Olsen et al., 1994) was implemented, which takes into account the transition/transversion ratio but does not allow for different base frequencies. When using PHYLIP the DNAML program was used (Felsenstein, 1981). The sequences were tested for recombination events or selection with a likelihood method implemented by PLATO (Grassly \& Holmes, 1997). In PLATO, the sequence alignment is scanned and each position is tested for the likelihood of producing the observed phylogenetic tree. Regions with sequence anomalies are given a score, the Z-value, where values over 3.5 are significant. A significant value of a certain region means that this region does not follow the phylogeny of the complete sequence, which could mean that a recombination event or unusually high selection pressure has occurred.

\section{RESULTS AND DISCUSSION}

\section{Sequencing and morphological observations}

In total, 31 nucleotide sequences from species of Nodularia were determined in this study. The nucleotide sequence of the partial (448 bp) het $R$ gene was determined for 16 Nodularia spp. strains. Out of these 16 strains 13 were also subjected to PCR and se- quencing of the PC-IGS (518 bp). For the remaining three strains (BC Nod 9401, BC Nod 9402 and BC Nod 9427) the PC-IGS sequence already existed (Hayes \& Barker, 1997). The het $R$ gene from nine strains of Aphanizomenon spp. and PC-IGS sequences from two Aphanizomenon spp. and A. lemmermanni KAC 16 were determined. The strains of Nodularia could be divided into two main groups based on their buoyancy. The $N$. harveyana KAC $34, N$. harveyana PCC 7804, N. spumigena KAC 32 and $N$. spumigena KAC 33 strains were all negatively buoyant. This group will be referred to as the non-floating group of strains. All the other Nodularia strains were floating. The het $R$ sequences from Aphanizomenon sp. originating from the Baltic Sea were compared with het $R$ sequences from the fresh-water strains of Aphanizomenon and the relatively closely related Anabaena spp. The morphology of Nodularia strains in culture varied between different strains and between different occasions of sampling of the identical strains (data not shown). The variation in morphology was such that no meaningful description of the strains in culture was possible. It has been shown that the morphology of Nodularia spp. is influenced by environmental and culturing conditions (Barker et al., 1999; Bolch et al., 1999; Lehtimäki et al., 2000). Indeed, the morphological characters of the strains presented here varied far more than the genetic distances would suggest. On the other hand, the sequences from $N$. spumigena isolates KAC 32 and KAC 33 were different from all other $N$. spumigena isolates. These differences could be due to inadequate identification, and highlight the problem of using morphology alone as a taxonomic criterion on cultured strains. Instead, a polyphasic approach including the organism's ecology, biochemical features and/or molecular phylogeny using mul- (a) hetR

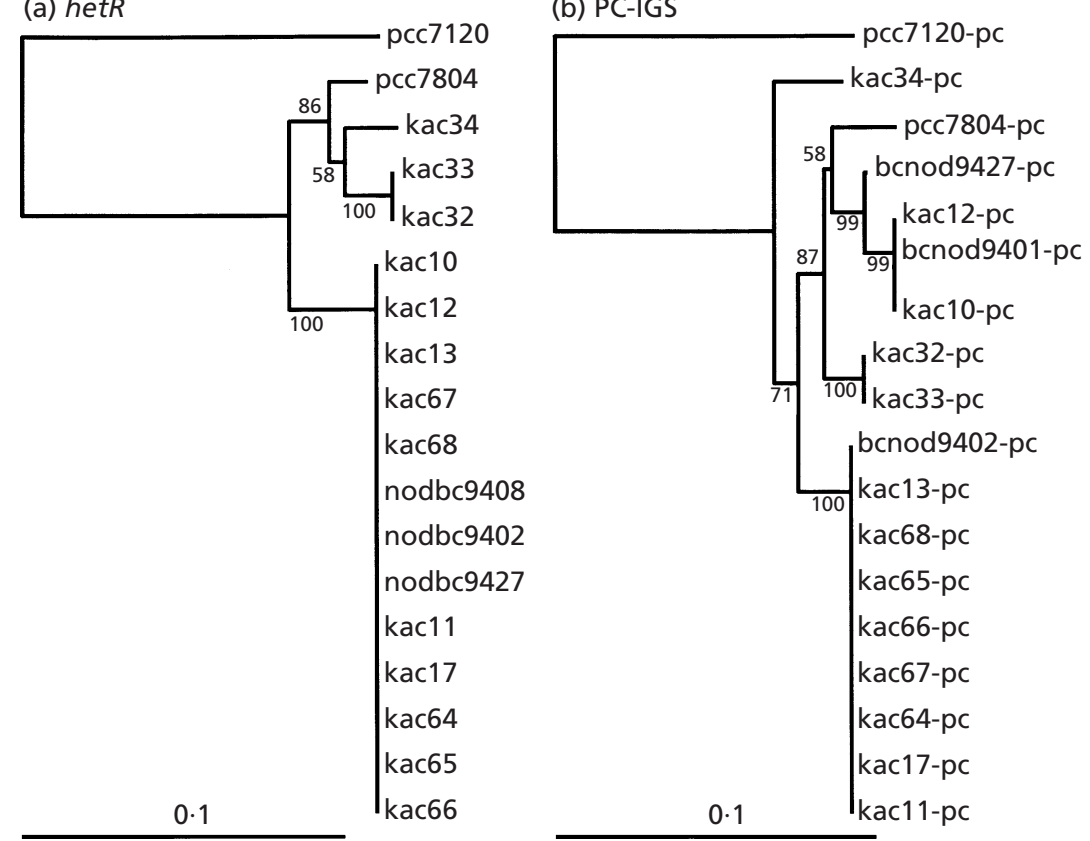

Fig. 1. Distance trees of sequences from two loci in Nodularia. The correction of the distance was that of Jukes \& Cantor (1969). The numbers at the branches are the bootstrap support as percentage of 500 replicates. The scales represent $10 \%$ sequence divergence. In (a), the tree was based on 448 positions of hetR sequences determined in this study, except pcc7120 and nodbc9408. In (b), the tree was inferred from 497 positions of PC-IGS sequences from the same strains as in (a). Note the different topologies between the two trees. 

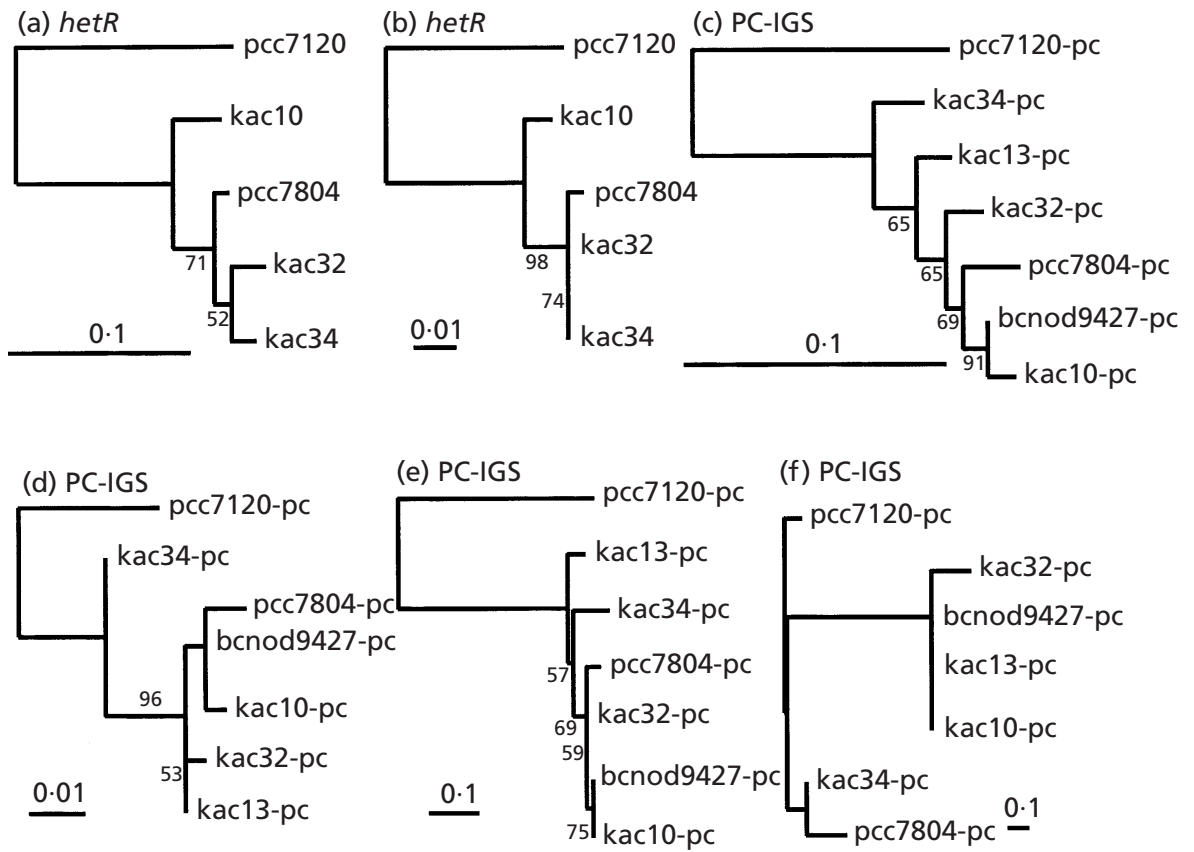

\begin{abstract}
Fig. 2. Comparison of maximum-likelihood phylogenies generated with various datasets of hetR and PC-IGS sequences from species of Nodularia. The numbers at branches and scales are as in Fig. 1, except that in (b) and (d) the scale represents $1 \%$ sequence divergence. In (a), the complete sequence was used and analysed with maximum-likelihood in PHYLIP using the observed transition/transversion ratio and base frequencies. In (b), the third position in the protein encoding frame has been removed from the analysis using PhyloWin. In (c), the complete sequence was used. In (d), the third position and the IGS region has been removed and (e) shows the tree based on the IGS region. In (f), the tree was based on the nucleotides 127-131 (422-426 in Nostoc sp. PCC 7120) that showed a significant sequence anomaly. The trees in (a) and (c) were generated with maximum-likelihood in PHYLIP using the observed transition/transversion ratio and base frequencies, all other trees were generated with PhyloWin.
\end{abstract}

tiple loci can better resolve species relationships among bacteria (Castenholtz, 1992; Ward, 1998).

\section{Phylogenetic trees of Nodularia sequences}

The overall relationship between the Nodularia strains and their het $R$ and PC-IGS sequences was displayed by inferring a distance tree (Fig. 1). The het $R$ phylogeny of the Nodularia spp. isolates clearly distinguished two main groups. All the floating strains had identical het $R$ sequences, which is in agreement with the suggestion that the pelagic population of Nodularia is represented by one species, N. spumigena (Barker et $a l ., 1999)$. In the sister group, were the het $R$ sequences from the non-floating strains PCC 7804, KAC 32, $\mathrm{KAC} 33$ and $\mathrm{KAC} 34$, where KAC 32 and KAC 33 were identical. In contrast, the tree of the PC-IGS sequences showed a different topology with some of the floating strains clustered together with non-floating strains (Fig. 1). The phylogenetic relationships was studied in more detail using a smaller dataset only taking into account those sequences that were different and using the maximum-likelihood approach to build the trees. The topology of the het $R$ tree using all positions gave the same topology as the first tree (Fig. 2a). In general, the third position in the derived reading frame of protein-encoding genes is more variable than the first and second. Reducing the dataset by using only the first and second positions of the sequences resulted in the same topology but the branch length between kac32 and kac34 became zero, i.e. they were identical (Fig. 2b). In the PC-IGS tree (Fig. 2c), as with the het $R$ tree, the topology of the tree did not change compared with the distance tree (Fig. 1b). The topology of the tree was the same when only the first and second positions were used, floating strains were still paraphyletic, but the branches became shorter (Fig. $2 \mathrm{~d})$. When only the intergenic spacer was used, in total 77 positions without gaps, the tree topology changed. The ancestral sequence was that of kac13-pc, as compared to kac34-pc in the previous trees, and the branch lengths became shorter and it was zero between kac10-pc and bcnod9427-pc (Fig. 2e).

Even though buoyancy and toxin production can be lost under culturing conditions, all the floating Nodularia $\mathrm{KAC}$-isolates are capable of producing the cyclic pentapeptide nodularin, a hepatotoxin that can also promote tumours (W. Stolte and E. Granéli, unpublished data; Dahlmann et al., 2001). The nonfloating $N$. harveyana PCC 7804 is capable of producing a nodularin of a different type, [L-Har $\left.{ }^{2}\right]$ nodularin, than those produced by $N$. spumigena (Beattie et $a l ., 2000)$. The PC-IGS phylogeny observed here and previously (Bolch et al., 1999) is not in accordance with the nodularin production as some of the sequences 
(a) hetR

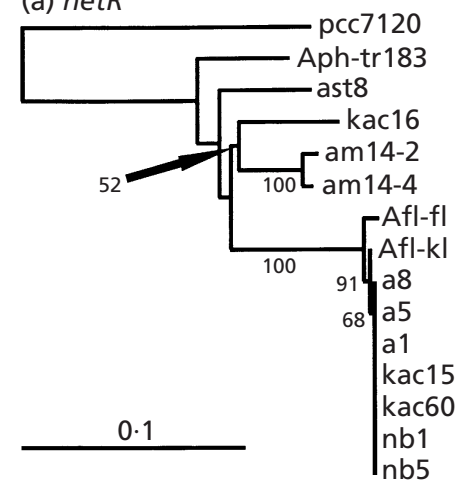

(b) PC-IGS

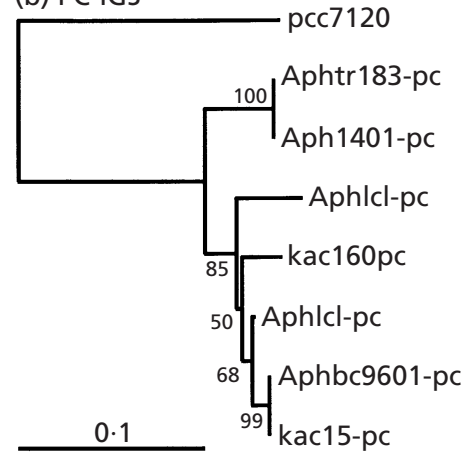

Fig. 3. Distance trees of sequences from two loci in a variety of Anabaena and Aphanizomenon species. The correction of the distance was that of Jukes \& Cantor (1969). The numbers at the branches are the bootstrap support as percentage of 500 replicates. The scales represent $10 \%$ sequence divergence. In (a), the tree was based on 448 positions of hetR sequences and in (b), on 343 positions of the PC-IGS locus. retrieved from the floating and toxin producing isolates clustered with sequences from the group of nonfloating isolates. A positive correlation with toxin production and $16 \mathrm{~S}$ rDNA sequences of $N$. spumigena has been reported previously (Lehtimäki et al., 2000; Moffit et al., 2001). These congruent results of $16 \mathrm{~S}$ rDNA and het $R$ suggest that the het $R$, but not the PCIGS phylogeny, was consistent with the organism phylogeny. The reason for the discrepancy between the relationships revealed by PC-IGS locus and the het $R$ / $16 \mathrm{~S}$ rDNA loci could result from gene transfer, as this has been suggested to occur among the different genotypes of $N$. spumigena in the Baltic Sea (Barker et al., 1999, 2000b).

\section{Phylogenetic trees of Anabaena and Aphanizomenon sequences}

The het $R$ sequences from Anabaena spp. and Aphanizomenon spp. were summarized in a distance tree (Fig. 3a). All the strains and natural populations of Aphanizomenon sp. from the Baltic Sea had zero branch length and formed a tight cluster with the two variants of Aphanizomenon flos-aquae with $100 \%$ bootstrap support. In contrast, the sequences from species of Anabaena showed multiple forms that suggested that several species exist in off-shore waters of the Baltic Sea. Aphanizomenon sp. TR183 was the earliest divergent sequence in this group. The PC-IGS sequences from identical, or morphologically similar, strains were summarized in a distance tree (Fig. 3b). The sequences from two isolates of Aphanizomenon sp. from the Baltic Sea had zero branch length, which is in accordance with the finding that the PC-IGS sequence from 25 colonies of Aphanizomenon sp. were identical (Barker et al., 2000a), while the Aphanizomenon sp. TR183 was identical to Aphanizomenon sp. CCAP 1401/1 and was ancestral to the other Anabaena/ Aphanizomenon sequences. The maximum-likelihood analysis of het $R$ sequences gave a tree similar to the distance tree except that the sequence from Aphanizomenon sp. TR183 was closest to the sequence of Anabaena lemmermannii KAC 16 (Fig. $4 a)$. When the first and second codon positions were used the Aphanizomenon sp. TR183 sequence formed a sister group to Anabaena lemmermannii KAC 16 (Fig. 4b). The branch length between the other Aphanizomenon strains was zero in the same tree. In the PC-IGS tree the topology changed when using maximumlikelihood and the bootstrap support was lower (Fig. $4 \mathrm{c})$. When the third position was removed from the analysis, the PC-IGS tree branching order remained unchanged, but with shorter branch lengths (Fig. 4d). The branching order changed again and the branch lengths were shorter when building the tree with the intergenic space including 71 positions (Fig. 4e). In this tree, the sequences of Aphanizomenon sp. from the Baltic Sea and Norman Lake were identical and ancestral to the sequences of Anabaena lemmermannii KAC 16, Aphanizomenon sp. from Little Crooked Lake, Aphanizomenon sp. TR183 and Aphanizomenon flos-aquae CCAP1401/1.

Based on ultrastructural observations the Aphanizomenon sp. from the Baltic Sea has been suggested to be a separate species from Aphanizomenon flos-aquae (Janson et al., 1994). Interestingly, the het $R$ sequence differed by $1.1 \%$ between these two organisms, which further suggests that they are not the same species. The sequences from Aphanizomenon flos-aquae var. klebahnii and Aphanizomenon sp. were almost identical, differing in only one nucleotide, which suggests a very close relationship between these two organisms. Surprisingly, the het $R$ sequence from the Baltic Sea isolate Aphanizomenon sp. TR183 was very different compared to the other Aphanizomenon spp. sequences with more than $11 \%$ sequence divergence. This strain has been reported to be inseparable from a cluster of Anabaena and Aphanizomenon strains based on the 16S rDNA phylogeny (Lyra et al., 2001). For example, the reported sequences of $16 \mathrm{~S}$ rDNA of Aphanizomenon sp. TR183 and Aphanizomenon sp. BC Aph 9601 differ by only $0 \cdot 1 \%$. Aphanizomenon sp. TR 183 has been used in physiological studies and inferences drawn in relation to the organisms forming blooms in the Baltic Sea (Evans et al., 2000), but it has been shown here that the cultured strain is not identical with the bloom-forming organism. Such data indicate that either the $16 \mathrm{~S}$ rDNA or the het $R$ sequence evolve at very different rates among different species of cyanobacteria. Consistent with the het $R$ phylogeny, the Aphanizomenon sp. TR183 PC-IGS sequence was 

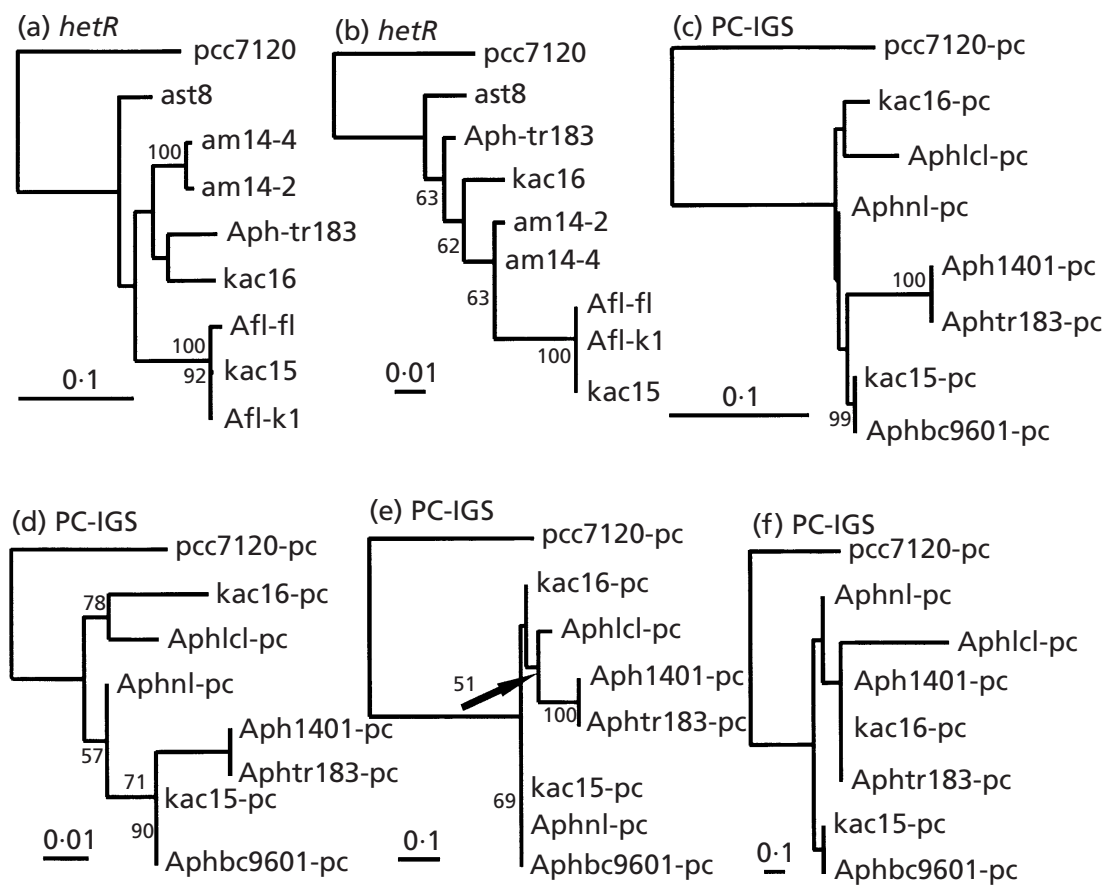

Fig. 4. Comparison of maximum-likelihood phylogenies generated with various datasets of hetR and PC-IGS sequences from species of Anabaena and Aphanizomenon. The numbers at branches and scales are as in Fig. 3, except that in (b) and (d) the scale represents $1 \%$ sequence divergence. In (a), the complete sequences of the hetR alleles have been used and in (b), the third position in the protein-encoding frame has been removed from the analysis. In (c), the complete sequences of the PC-IGS alleles were used and in (d) the third position and the IGS region were removed. In (e), the IGS region was included only and in (f), the tree based on the nucleotides 116-128 (411-423 in Nostoc sp. PCC 7120) showed a significant sequence anomaly. The trees in (a) and (c) were generated with maximum-likelihood in PHYLIP using the observed transition/transversion ratio and base frequencies; all other trees were generated with PhyloWin.

clearly separated from the sequence of Aphanizomenon sp. KAC 15 in all trees. Moreover, it did not form macrocolonies upon bubbling the culture, as did the Aphanizomenon sp. KAC 15 (data not shown). Taken together the simplest way to explain these observed differences between 16S rDNA phylogenies on the one hand and het $R / \mathrm{PC}-\mathrm{IGS}$ on the other is that $16 \mathrm{~S}$ rDNA displays an extremely slow evolution in the observed taxa, which makes the 16S rDNA sequence unsuitable for resolving relationships between species of heterocystous cyanobacteria.

\section{Analysis of sequence anomalies}

In order to assess the possibility of sequence biases within the loci used in this study, the sequences were tested with PLATO. This test showed that no sequence anomalies were detectable in the het $R$ sequences. In contrast, the PC-IGS sequences from both Nodularia spp. and Anabaena/Aphanizomenon gave significant values in this test. The tree that was tested against the alignment was that in Figs 2(a, c) and 4(a, c), and the parameters given to the program were the model (Hasegawa et al., 1985) and the transition/transversion value of 0.916 and 0.99 . The sequences from species of Nodularia gave a Z-value of $8 \cdot 1$ for the region between position 239 and 288 (corresponds to the intergenic spacer excluding 36 nucleotides at the $3^{\prime}$ end). A Z-value above 3.6 was significant in this analysis. A second region also gave a significant Zvalue of $3 \cdot 7$, positions 127-131 (422-426 in Nostoc sp. PCC 7120), which is within the $c p c B$ coding region. The phylogeny of this region differs from that seen when all positions were used (Fig. 2c, f). The differences also indicate that the amino acid isoleucine 142 in Nostoc sp. PCC 7120 is unchanged in N. harveyana KAC 34 but had changed to leucine in all the other strains. Significant Z-values in the Anabaena/Aphanizomenon sequences were those above $3 \cdot 5$. Positions 234-272 and 304-315, which are within the intergenic spacer, gave Z-values of 4.8 and $3 \cdot 5$, respectively. Positions 116-128 in Anabaena/Aphanizomenon (411423 in Nostoc sp. PCC 7120), which are within the coding region of $c p c B$, gave a $\mathrm{Z}$-value of 4.4 . The phylogeny of the Anabaena/Aphanizomenon group of sequences also differed from the phylogeny seen when using all positions (Fig. 4c, f). Here, the indicated amino acid change was glycine 141 in Nostoc sp. PCC 7120, which was unchanged in Aphanizomenon sp. KAC 15, Aphanizomenon sp. BC Aph 9601 and Aphanizomenon sp. Norman Lake derived sequences but which had changed to alanine in all other strains (data not shown).

The complexity of the evolution of the PC-IGS alleles observed in the Baltic Sea has been attributed to 
horizontal gene transfer (Barker et al., 2000b). This alone should make this locus less suitable for phylogenetic analysis. In addition, we have shown that in two different groups of heterocystous cyanobacteria, the intergenic space shows less variation than the coding region and that it has a different phylogeny from the coding region. This is surprising because intergenic spaces are generally considered to be more variable than the coding region and this indicates that there has been either selection or recombination events within the PC-IGS region.

\section{Concluding remarks}

The phylogeny of the PC-IGS locus must be treated with much care when attempting to deduce organismal phylogenies as the locus displays a complex evolutionary pattern. In contrast, it has been shown by this and previous studies that the het $R$ gene phylogeny seem to closely match the organism phylogeny, and there are no detectable selection biases within the partial het $R$ gene sequences presented here. This study also confirms the suggestions that $N$. spumigena is the only Nodularia species in the Baltic Sea and that the Baltic Sea Aphanizomenon sp. is not identical with Aphanizomenon flos-aquae found in lakes.

\section{ACKNOWLEDGEMENTS}

We are grateful to Christina Esplund for maintaining all cultures used in this study. We thank Dr Susanna Hajdu, Department of Systems Ecology, Stockholm University, for sending samples from the Landsort deep and Dr Gertrud Cronberg, Department of Ecology, University of Lund, for providing samples from lake Bysjön, and Lilla Pildammen. Drs Kaarina Sivonen and Jaana Lehtimäki, Department of Applied Chemistry and Microbiology, University of Helsinki, who generously provided Aphanizomenon sp. TR 183. Dr Gary Barker and Paul Hayes, School of Biological Sciences, University of Bristol, for providing DNA from their Nodularia isolates. The authors also thank Dr Jeff Elhai at the Department of Biology, University of Richmond, for critically reviewing an early draft of this manuscript. This research was supported by the EC Marine Science and Technology programme under contract MAS3CT97-0149 (DOMTOX).

\section{REFERENCES}

Barker, G. L. A., Hayes, P. K., O'Mahony, S. L., Vacharapiyasophon, P. \& Walsby, A. E. (1999). A molecular and phenotypic analysis of Nodularia (Cyanobacteria) from the Baltic Sea. J Phycol 35, 931-937.

Barker, G. L. A., Konopka, A., Handley, B. A. \& Hayes, P. K. (2000a). Genetic variation in Aphanizomenon (cyanobacteria) colonies from the Baltic Sea and North America. J Phycol 36, 947-950.

Barker, G. L. A., Handley, B. A., Vacharapiyasophon, P., Stevens, J. R. \& Hayes, P. K. (2000b). Allele-specific PCR shows that genetic exchange occurs among genetically diverse Nodularia (Cyanobacteria) filaments in the Baltic Sea. Microbiology 146, 2865-2875.

Beattie, K. A., Kunimitsu, K. \& Codd, G. A. (2000). The cyanobacterium Nodularia PCC 7804, of freshwater origin, produces [L-Har $\left.{ }^{2}\right]$ nodularin. Phytochemistry 54, 57-61.

Bolch, C. J. S., Orr, P. T., Jones, G. J. \& Blackburn, S. I. (1999). Genetic, morphological and toxicological variation among globally distributed strains of Nodularia (Cyanobacteria). J Phycol 35, 339-355.
Buikema, W. J. \& Haselkorn, R. (1991). Characterization of a gene controlling heterocyst differentiation in the cyanobacterium Anabaena 7120. Genes Dev 5, 321-330.

Buikema, W. J. \& Haselkorn, R. (2001). Expression of the Anabaena het $R$ gene from a copper-regulated promoter leads to heterocyst differentiation under repressing conditions. Proc Natl Acad Sci U S A 98, 2729-2734.

Capone, D. G., Zehr, J. P., Paerl, H. W., Bergman, B. \& Carpenter, E. J. (1997). Trichodesmium, a globally significant marine cyanobacterium. Science 276, 1221-1229.

Carpenter, E. J., Montoya, J. P., Burns, J., Mulholland, M. R., Subramaniam, A. \& Capone, D. G. (1999). Extensive bloom of an $\mathrm{N}_{2}-$ fixing diatom/cyanobacterial association in the tropical Atlantic Ocean. Mar Ecol Prog Ser 185, 273-283.

Castenholz, R. W. (1992). Species usage, concept, and evolution in the cyanobacteria (blue-green algae). $J$ Phycol 28, 737-745.

Dahlmann, J., Rühl, A., Hummert, C., Liebezeit, G., Carlsson, P. \& Granéli, E. (2001). Different methods for toxin analysis in the cyanobacterium Nodularia spumigena (Cyanophyceae). Toxicon 35, 1183-1190.

Evans, A. M., Gallon, J. R., Jones, A., Staal, M., Stal, L. J., Villbrandt, M. \& Walton, T. J. (2000). Nitrogen fixation by Baltic cyanobacteria is adapted to the prevailing photon flux density. New Phytol 147, 285-297.

Felsenstein, J. (1981). Evolutionary trees from DNA sequences: a maximum likelihood approach. J Mol Evol 17, 368-376.

Fox, G. E., Wisotzkey, J. D. \& Jurtshuk, P., Jr (1992). How close is close: $16 \mathrm{~S}$ rRNA sequence identity may not be sufficient to guarantee species identity. Int J Syst Bacteriol 42, 166-170.

Galtier, N., Gouy, M. \& Gautier, C. (1996). SEAVIEW and PHYLO_WIN: two graphic tools for sequence alignment and molecular phylogeny. Comput Applic Biosci 12, 543-548.

Gantt, E. (1994). Supramolecular membrane organization. In The Molecular Biology of Cyanobacteria, pp. 119-138. Edited by D. A. Bryant. Dordrecht: Kluwer Academic.

Geitler, L. (1932). Cyanophyceae. In Rabenhorst's Kryptogamenflora von Deutschland, Österreich und der Schweiz. Edited by R. Kolkwitz. Leipzig: Akademische Verlagsgesellschaft.

Grassly, N. C. \& Holmes, E. C. (1997). A likelihood method for the detection of selection and recombination using sequence data. Mol Biol Evol 14, 239-247.

Hasegawa, M., Kishino, H. \& Yano, T. (1985). Dating the human-ape splitting by a molecular clock of mitochondrial DNA. J Mol Evol 22, 160-174.

Hayes, P. K. \& Barker, G. L. A. (1997). Genetic diversity within Baltic Sea populations of Nodularia (Cyanobacteria). J Phycol 33, 919-923.

Janson, S., Carpenter, E. J. \& Bergman, B. (1994). Fine structure and localisation of proteins in Aphanizomenon sp. from the Baltic Sea. Eur J Phycol 29, 203-211.

Janson, S., Matveyev, A. \& Bergman, B. (1998). The presence and expression of hetR in the non-heterocystous cyanobacterium Symploca PCC 8002. FEMS Microbiol Lett 168, 173-179.

Janson, S., Bergman, B., Carpenter, E. J., Giovannoni, S. J. \& Vergin, K. (1999a). Genetic analysis of natural populations of the marine diazotrophic cyanobacterium Trichodesmium. FEMS Microbiol Ecol 30, 57-65.

Janson, S., Wouters, J., Bergman, B. \& Carpenter, E. J. (1999b). Host specificity in the Richelia-diatom symbiosis revealed by het $R$ gene sequence analysis. Environ Microbiol 1, 431-438.

Jukes, T. H. \& Cantor, C. R. (1969). Evolution of protein molecules. In Mammalian Protein Metabolism, pp. 21-132. Edited by H. N. Munro. New York: Academic Press.

Komárek, K., Hübel, M., Hübel, H. \& Smarda, J. (1993). The Nodularia studies 2. Taxonomy. Arch Hydrobiol (Suppl 96)/Algol Stud 68, $1-25$.

Lehtimäki, J., Lyra, C., Suomalainen, S., Sundman, P., Rouhiainen, L., Paulin, L., Salkinoja-Salonen, M. \& Sivonen, K. (2000). Charac- 
terization of Nodularia strains, cyanobacteria from brackish waters, by genotypic and phenotypic methods. Int J Syst Evol Microbiol 50, 1043-1053.

Leppänen, J.-M., Niemi, Å. \& Rinne, I. (1988). Nitrogen fixation of cyanobacteria (blue-green algae) and the nitrogen cycle of the Baltic sea. Symbiosis 6, 181-194.

Li, W.-H. \& Graur, D. (1991). Fundamentals of molecular evolution. Sunderland, MA: Sinauer Associates.

Lyra, C., Suomalainen, S., Gugger, M., Vezie, C., Sundman, P., Paulin, L. \& Sivonen, K. (2001). Molecular characterization of planktic cyanobacteria of Anabaena, Aphanizomenon, Microcystis and Planktothrix genera. Int J Syst Evol Microbiol 51, 513-526.

Moffitt, M. C., Blackburn, S. I. \& Neilan, B. A. (2001). rRNA sequences reflect the ecophysiology and define the toxic cyanobacteria of the genus Nodularia. Int J Syst Evol Microbiol 51, 505-512.

Neilan, B. A., Jacobs, D. \& Goodman, A. E. (1995). Genetic diversity and phylogeny of toxic cyanobacteria determined by DNA polymorphisms within the phycocyanin locus. Appl Environ Microbiol 61 3875-3883.

Olsen, G. J., Matsuda, H., Hagstrom, R. \& Overbeek, R. (1994). fastDNAml: a tool for construction of phylogenetic trees of DNA sequences using maximum likelihood. Comput Appl Biosci 10, 41-48.
Rahm, L., Jönsson, A. \& Wulff, F. (2000). Nitrogen fixation in the Baltic proper: an empirical study. J Mar Sys 25, 239-248.

Rippka, R. (1988). Isolation and purification of cyanobacteria. Methods Enzymol 167, 3-27.

Robertson, B. R., Tezuka, N. \& Watanabe, M. M. (2001). Phylogenetic analyses of Synechococcus strains (cyanobacteria) using sequences of $16 \mathrm{~S}$ rDNA and part of the phycocyanin operon reveal multiple evolutionary lines and reflect phycobilin content. Int J Syst Evol Microbiol 51, 861-871.

Tillett, D., Parker, D. L. \& Neilan, B. A. (2001). Detection of toxigenicity by a probe for the microcystin synthetase A gene (mcyA) of the cyanobacterial genus Microcystis: comparison of toxicities with $16 \mathrm{~S}$ rRNA and phycocyanin operon (phycocyanin intergenic spacer) phylogenies. Appl Environ Microbiol 67, 2810-2818.

von Wintzingerode, F., Göbel, U. B. \& Stackebrandt, E. (1997). Determination of microbial diversity in environmental samples: pitfalls of PCR-based rRNA analysis. FEMS Microbiol Rev 21, 213-229.

Ward, D. M. (1998). A natural species concept for prokaryotes. Curr Opin Microbiol 1, 271-277.

Zhou, R., Wei, X., Jiang, N., Li, H., Dong, Y., Hsi, K.-L. \& Zhao, J. (1998). Evidence that HetR protein is an unusual serine-type protease. Proc Natl Acad Sci U S A 95, 4959-4963. 